\title{
émulations
}

\section{Regards croisés de mères, de donneurs et d'enfants de familles lesboparentales en France}

\author{
Isabel Côté, Martine Gross, Kévin Lavoie, Line Chamberland
}

Émulations - Revue de sciences sociales

2019, n 32, « Aux frontières de la parenté. Un éclairage par les marges ».

\section{Article disponible à l'adresse suivante}

https://ojs.uclouvain.be/index.php/emulations/article/view/coteetal

\section{Pour citer cet article}

Isabel Côté, Martine Gross, Kévin Lavoie, Line Chamberland, « Regards croisés de mères, de donneurs et d'enfants de familles lesboparentales en France », Émulations, $\mathrm{n}^{\circ}$ 32, Mise en ligne le 20 avril 2020.

DOI : 10.14428/emulations.032.07

Distribution électronique : Université catholique de Louvain (Belgique) : ojs.uclouvain.be

(C) Cet article est mis à disposition selon les termes de la Licence Creative Commons Attribution, Pas d'Utilisation Commerciale 4.0 International. http://creativecommons.org/licenses/by-nc/4.0/

Éditeur : Émulations - Revue de sciences sociales / Presses universitaires de Louvain https://ojs.uclouvain.be/index.php/emulations

ISSN électronique : 1784-5734

$\frac{\text { PUL PRESSES }}{\text { UNIVERSITAIRES }}$




\title{
Regards croisés de mères, de donneurs et d'enfants de familles lesboparentales en France ${ }^{1}$
}

\author{
Isabel Côté2, Martine Gross³, Kévin Lavoie ${ }^{4}$, \\ Line Chamberland ${ }^{5}$
}

\begin{abstract}
[Résumé] En France, la plupart des mères lesbiennes se tournent vers la procréation médicalement assistée (PMA) pour fonder une famille. Un certain nombre d'entre elles ont cependant recours à un "donneur connu », c'est-à-dire un homme de leur entourage qui accepte de faire un don pour les aider à devenir mères sans pour autant revendiquer une paternité légale. Cet article explore cette configuration familiale en documentant les relations entretenues entre les mères lesbiennes, leur donneur, mais également les enfants issus de ses dons. Quatre rôles endossés par le donneur se dégagent en fonction de la proximité établie avec les mères et de la relation entretenue ou non avec l'enfant au fil du temps : 1) une " quasi » coparentalité planifiée ; 2) un donneur considéré comme un père par une partie de la famille ; 3 ) un donneur " proche » ou 4) un donneur « distant», voire inconnu.
\end{abstract}

Mots clés : famille lesboparentale, mère, donneur, enfant, relations familiales, France.

\section{Crossed views of mothers, donors and children of lesbian families in France}

[Abstract] In France, most lesbian mothers turn to medically assisted reproduction (MAP) to start a family. However, a number of them use a "known donor", i.e. a close male who agrees to make a sperm donation to help them to become mothers without claiming legal paternity. This paper explores this family configuration by documenting the relationships between lesbian mothers, their donors, but also the children born from their donations. Four roles for the donor emerge depending on the proximity established with the mothers and the relationship with the child over time: 1) a "quasi" co-parent relationship; 2) a donor considered as a father by a part of the family; 3) a "close" donor or 4) a "distant" donor, or even unknown.

Keywords: lesbian families, mother, donors, children, family relationships, France.

Après des débats houleux, la loi adoptée le 17 mai 2013 autorise désormais les couples de même sexe français à se marier. Cette ouverture sur le plan matrimonial a aussi permis une reconnaissance juridique des familles lesboparentales, mais sous certaines

\footnotetext{
${ }^{1}$ L'équipe de recherche Savoirs sur l'inclusion et l'exclusion des personnes LGBTQ (SAVIE-LGBTQ) tient à remercier toutes les personnes qui ont participé à la recherche et ont confié leurs expériences à SAVIE-LGBTQ. Nous remercions également l'équipe complète des cochercheur·e·s et collaborateurs·trice·s, des organismes partenaires et des représentant·e·s d'organismes associés à la recherche. La recherche SAVIE-LGBTQ a été rendue possible grâce au financement du Conseil de recherches en sciences humaines du Canada (CRSH) et aux contributions des partenaires et autres organismes contributeurs au projet.

2 Université du Québec en Outaouais, département de travail social, Canada.

${ }^{3}$ Centre national de la recherche scientifique, CESOR, France.

${ }^{4}$ Université de Laval, École de travail social et de criminologie, Canada.

${ }^{5}$ Université du Québec à Montréal, département de sexologie, Canada.
} 
conditions : les deux femmes doivent être mariées, et celle n'ayant pas porté son enfant doit s'astreindre à un processus d'adoption pour obtenir les mêmes responsabilités et droits parentaux que son épouse qui a donné naissance (Brunet, 2015). L'adoption intraconjugale est alors une pratique utilisée pour sécuriser la filiation de l'enfant avec sa mère jusqu'alors non statutaire. D’autres embûches jalonnent l'accès à la maternité lesbienne en France, puisque l'assistance médicale à la procréation (AMP) est réservée aux couples hétérosexuels. Exclus des centres de procréation assistée, les couples lesbiens doivent franchir les frontières et se rendre dans un autre pays, en Belgique ou en Espagne par exemple, pour recourir à une insémination artificielle avec donneur.

Si la plupart des lesbiennes françaises se tournent vers l'AMP avec don anonyme pour devenir mères (Gross, Courduriès, deFederico, 2014a), un certain nombre d'entre elles ont cependant recours à un " donneur connu ", c'est-à-dire un homme de leur entourage qui accepte de faire un don pour les aider à fonder une famille, sans pour autant devenir le père légal de l'enfant. Dans l'enquête nationale sur le fonctionnement conjugal et familial des familles homoparentales menée en 2012 auprès de 409 mères lesbiennes, $14 \%$ avaient eu recours à un tel don (Gross, Courduriès, deFederico, 2014b). Or, en France, contrairement au contexte québécois par exemple (Leckey, 2014), cet homme a la possibilité de revendiquer sa paternité tant que l'adoption de l'enfant par la conjointe de la mère n'a pas été prononcée, empêchant dès lors l'établissement de la double filiation maternelle. Si les mères peuvent craindre que le donneur change d'avis et reconnaisse l'enfant issu de son don, lui-même n'est pas protégé juridiquement tant que l'adoption intraconjugale n'a pas été prononcée. En effet, les mères peuvent poursuivre le donneur pour faire établir sa filiation paternelle et éventuellement obtenir des subsides pendant la minorité de l'enfant (ou l'enfant lui-même jusqu'à ses 28 ans). Les motivations avancées pour recourir à un donneur connu en France en dehors de tout cadre légal tiennent au souhait de pouvoir renseigner l'enfant sur ses origines, mais aussi pour éviter un parcours médicalisé ou encore, pour des raisons économiques.

Des recherches empiriques menées dans d'autres contextes socio-législatifs ont permis de mieux comprendre la relation qui unit les mères lesbiennes à leur donneur ou encore, celle de ces enfants avec l'homme à l'origine de leur naissance. Au regard du contexte québécois, Côté et Lavoie (2016) ont démontré que les liens entre les femmes et leur donneur varient selon leurs attentes respectives et sont susceptibles d'évoluer à travers le temps. Le rôle endossé par cet homme au sein de la famille lesboparentale s'ancre dans des représentations plurielles de la parenté, lesquelles ont été conceptualisées par Côté (2014) sur un continuum allant d'un « donneur géniteur » à un « donneur père », en passant par un " donneur périphérique ». Chacune de ces figures incarne un niveau différencié d'implication et de proximité relationnelle, lesquelles sont négociées entre les parties préalablement au don (Côté, Lavoie, 2016). Des constats similaires ont été observés ailleurs dans le monde, par exemple en Grande-Bretagne (Donovan, 2000), en Australie (Dempsey, 2012) et en Nouvelle-Zélande (Surtees, 2017). Quant aux enfants des familles lesboparentales, rares sont les études qui se sont attachées à com- 
prendre les liens qu'ils entretiennent avec le donneur (Côté et al., 2019; Malmquist et al., 2014). Ces recherches démontrent que les enfants concernés font une nette distinction entre la paternité biologique et la paternité sociale et que conséquemment, si un donneur peut être un père, il ne peut pas être un « papa » à moins d'être impliqué dans les activités de parentage.

Bien que pertinents pour comprendre les relations qui unissent les personnes concernées, ces travaux ne se basent généralement que sur un seul point de vue, ou combinent uniquement celui des mères et des donneurs (Golombok, 2015 ; Nordqvist, 2014). À notre connaissance, aucune recherche n’a jusqu'à maintenant croisé les regards de l'ensemble des membres de la triade dans le but de cerner les frontières de la parenté en contexte français. Cet article vise à explorer cette configuration familiale lesboparentale en documentant les relations entretenues entre les mères et leur donneur, mais également entre ce dernier et les enfants issus de ses dons.

\section{Présentation de la recherche}

Notre enquête de terrain a été menée entre 2017 et 2018, période pendant laquelle nous avons rencontré 49 personnes. Notre analyse s'appuie sur des entrevues qualitatives conduites avec 11 familles lesboparentales françaises comprenant 21 mères (neuf couples, deux mères séparées qui avaient les enfants en résidence alternée et une mère célibataire) et 15 enfants âgés de 5 à 14 ans. Les femmes que nous avons rencontrées étaient âgées en moyenne de 45 ans. La plus âgée avait 53 ans, la plus jeune 33 ans. Trois personnes étaient en recherche d'emploi ; les autres travaillaient pour la plupart à temps plein. Hormis deux ouvrières ou employées, toutes celles qui travaillaient exerçaient des professions de catégories moyennes ou supérieures. Les revenus de la famille atteignaient au total en moyenne 3316 euros mensuels. Le maximum étant de 7800 euros et le minimum 2100. Leur niveau éducatif était en moyenne de Bac+4, le diplôme le moins élevé était le baccalauréat et le diplôme le plus élevé un doctorat. Nous avons également rencontré neuf donneurs et quatre partenaires de vie de ces hommes.

Les donneurs étaient âgés en moyenne de 40 ans. Le plus jeune était âgé de 28 ans et le plus âgé de 78 ans. Leur niveau éducatif est aussi assez élevé puisque la moyenne est de bac+4. Le diplôme le moins élevé était un $\mathrm{CAP}^{6}$. Le diplôme le plus élevé était un doctorat. Nous n'avons pas les données socio-démographiques des partenaires de vie. Dans trois situations familiales, nous n'avons pas pu rencontrer les donneurs ; dans un cas parce que les mères n'avaient aucun contact avec lui, dans l'autre parce qu'il a refusé de nous accorder un entretien. Enfin, dans l'une des familles, le donneur rencontré est celui de la cadette, âgée de quelques mois, et non pas celui de l'aînée qui a participé à la présente recherche.

Les familles ont été recrutées principalement via des associations qui ont accepté de diffuser une annonce auprès des familles homoparentales adhérentes. Des annonces ont également été publiées en ligne sur des pages Facebook spécifiques au don

\footnotetext{
${ }^{6}$ Certificat d'aptitude professionnelle.
} 
de sperme. Nous avons tout d'abord rencontré les mères, qui nous ont ensuite mis en contact avec leur donneur tout en autorisant également notre équipe à rencontrer leurs enfants. Pour les entrevues conduites auprès des adultes, nous avons utilisé l'approche compréhensive telle que développée par Jean-Claude Kaufmann (2011). Chaque personne rencontrée était alors considérée comme une représentante de sa catégorie (mères, donneurs ou partenaires de vie de ces derniers) permettant de croiser les expériences et ainsi construire une compréhension globale du phénomène à l'étude. Pour ce faire, nous avons mené des entretiens semi-directifs approfondis pour appréhender les expériences des membres de la triade selon le sens qu'ils et elles donnent à leurs actions.

L'approche centrée sur l'enfant (child-centered approach) a guidé les entrevues avec nos jeunes participants. Selon cette perspective, les enfants développent leur propre compréhension des liens entre les membres d'une famille et possèdent une créativité et une flexibilité particulières qui leur permettent de réfléchir de façon singulière aux relations familiales complexes (Mason, Tipper, 2008). La parole de l'enfant contribue donc à une triangulation des données, ce qui permet de nuancer et d'augmenter la validité des résultats obtenus auprès des adultes (Dubeau, Coutu, Tremblay, 2008). Cela apparait particulièrement porteur pour mieux comprendre la déliaison des liens sociaux, juridiques et biologiques chez les familles lesboparentales, où les frontières de la parenté sont mouvantes. Pour maximiser l'apport de l'enfant dans le processus de recherche et favoriser sa réflexion sur les liens entre apparentés, nous avons utilisé la cartographie circulaire (Samuelson, Thernlund, Ringström, 1996), outil validé ayant fait ses preuves dans des recherches auprès d'enfants grandissant dans des structures familiales variées (Côté et al., 2019 ; Roe, Bridges, Dunn, O’Connor, 2006).

Pour maximiser la liberté de parole et assurer la confidentialité des échanges, les membres de chaque unité familiale - les mères, les donneurs et les enfants - ont été rencontrés individuellement. Ce choix apparait d'autant plus important que la validité des données recueillies auprès de l'ensemble des protagonistes exige une étanchéité absolue de telle sorte que chacun puisse se sentir libre de se confier à l'équipe de recherche. L'étude a obtenu l'approbation du comité éthique de la recherche de l'Université du Québec en Outaouais (Canada).

\section{Résultats}

Pour toutes les personnes rencontrées, les parents sont les mères. Sauf dans un cas, les donneurs ne prennent pas part à l'éducation de l'enfant issu de leur don. Quant à leur place dans la configuration familiale ou leur rôle auprès de l'enfant, on peut entrevoir quatre types de position en fonction de la distance établie avec le couple lesboparental ou de la relation avec l'enfant : 1) une « quasi » coparentalité planifiée ; 2) un donneur considéré comme un père par une partie de la famille ; 3) un donneur « proche » ou 4) un donneur « distant », voire inconnu. 


\section{1. «Quasi » coparentalité planifiée}

Dans la situation de « quasi » coparentalité planifiée ${ }^{7}$ qui concerne une seule famille, le donneur, même s'il n'a pas le statut légal de père, se considère lui-même comme un père et est perçu comme tel par les mères et l'enfant, qui l'appelle " papa ». Il voit très régulièrement l'enfant et participe à son éducation, que ce soit parce que les mères le consultent pour plusieurs des décisions, ou encore, parce qu'il intervient sur le plan disciplinaire. Il s'agit d'une organisation familiale particulière alors que Léo ${ }^{8}$, le donneur, est le mari de la sœur de l'une des mères. S'il participe à l'éducation de l'enfant, ce sont cependant les deux mères d’Éliane (neuf ans) qui sont légalement reconnues comme ses parents. Son implication auprès de la fillette a été convenue dès le départ, facilitée en cela par la qualité de la relation unissant les deux couples. Léo est également le père de Nathan (sept ans). Les deux enfants sont conscients des liens qui les unissent, puisque les discussions à ce sujet sont transparentes, comme en témoigne Léo : "Ils sont au courant de la situation. Je suis le papa. Ils s'appellent "petit frère, grande sœur”. Pour eux, il n'y a aucun problème là-dessus. On leur a expliqué clairement et simplement avec des mots qu'ils peuvent comprendre, comment ça se fait que j'aie un enfant avec les deux sœurs. Les enfants n'ont jamais eu l'air embêtés avec ça ».

Interrogés sur leur système familial, les deux enfants présentent le même discours : "Quand on me pose la question, je dis que j'ai deux mamans qui s'aiment et elles ont voulu un enfant, explique Éliane, la fille aînée. Elles ont demandé à mon père s'il était d'accord, et voilà ». Nathan, le cadet, précise : "En fait, c'est que mon père, il s'est marié avec ma maman, mais vu qu'elles étaient mariées les deux tatas, il a fait un bébé avec une de mes tatas. Ça fait qu'on est demi-frère et demi-sœur ». Malgré cette compréhension commune des liens qui les unissent l'un à l'autre, les deux enfants circonscrivent leur système familial aux parents qui en prennent soin dans leur vie de tous les jours, à savoir ses deux mères pour Éliane, et son père et sa mère pour Nathan. Tous deux s'appliquent alors à tracer une frontière. Questionné sur ce qu'est une demisœur, Nathan affirme : «C'est une sœur et une cousine », opérant dès lors une certaine distance avec elle. Quant à Éliane, elle explique : «Ma maman Emmanuelle, ce n’est pas totalement quelqu'un de la famille de mon frère », histoire de bien départager les deux unités familiales.

\footnotetext{
${ }^{7}$ Contrairement à la coparentalité post-rupture qui s'instaure après une séparation parentale, la coparentalité planifiée survient lorsque des personnes ne partageant pas une relation conjugale décident de mettre en commun leur désir d'enfant et de fonder une famille (Herbrand, 2018). Par exemple, un couple lesbien et un couple gai deviennent parents d'un enfant duquel elles et ils auront la garde partagée. Si trois personnes ou plus sont impliquées dans le projet coparental, seul deux d'entre elles pourront être reconnues légalement comme parents, conformément à l'état actuel du droit en France.

${ }^{8}$ Pour des raisons d'anonymisation, tous les prénoms ont été remplacés.
} 


\subsection{Donneur considéré comme un père par une partie de la famille}

Dans ce deuxième cas qui concerne deux situations familiales, il a été convenu, lors des discussions préalables au don, que le donneur ne serait pas présenté à l'enfant comme son père. De ce fait, il n'aurait aucun droit ni devoir normalement dévolus à la paternité. Cependant, après la naissance de l'enfant, une partie des protagonistes considèrent le donneur comme un père, le désignent comme tel et expriment des attentes à son égard. Dans cette situation, le donneur rencontre régulièrement l'enfant issu de son don.

Éric, lui-même père de deux enfants, était le mari d'une amie proche du couple formé de Nadine et Caroline, aujourd'hui séparées. Au départ, il était convenu qu'Éric ne reconnaisse pas l'enfant et ne s'implique pas dans son éducation; la naissance de l'enfant résultant d'un projet parental que seules les mères avaient formulé. Avant que Nadia (14 ans) n'atteigne l'âge de huit ou neuf ans, jamais Éric n'était désigné comme le père. Il était simplement « Éric » ou « le géniteur ». Ce n'est que plus tard que le mot « père » a commencé à être utilisé par Nadia et sa mère. Par souci, notamment, d'aider l'enfant à intégrer la question de ses origines. Éric a alors accepté cette appellation : «C'était inconcevable pour moi de participer à la conception d'un être sans que cet être n'ait le moyen de remonter à son géniteur, si vous voulez, à son "père", non pas dans le sens "père" du terme, mais dans le sens "géniteur" du terme. De savoir ses gènes, son ascendant ». Quand on lui demande combien il a d'enfants, il répond : « Je dis que j’ai trois enfants, ou alors je dis que j'en ai deux, plus une ». Sa définition de la paternité s'attache à la dimension génétique, mais sa perception demeure ambiguë : il se sent des obligations, mais pas de responsabilités. Il a noué avec Nadia une profonde relation affective.

Cette relation affective est partagée par Nadia, bien que les contacts entre elle et Éric soient plutôt rares. L'absence de quotidienneté amène d'ailleurs l'adolescente à privilégier le terme de " père » et non celui de " papa » pour le désigner : "C'est mon père, je l'appelle par son prénom. Il compte beaucoup pour moi, mais vu qu'il n'est pas très présent dans ma vie - dans mon cœur, beaucoup - ce n'est pas un papa, c'est plus mon géniteur. [...] Moi, dans ma situation, il ne m'élève pas. Il n'a pas un rôle de père qui éduque son enfant, qui lui donne de l'amour tous les jours ».

Nadine, la mère de Nadia, si elle encourage les relations entre sa fille et Éric, témoigne quand même du fait que cette paternité n'implique aucun engagement formel. En ce sens, elle fait une nette distinction entre la relation que Nadia entretient avec son père de celle qu'elle a elle-même eue avec le sien. Cela s'explique par le fait que les relations ne sont pas très soutenues, l'enfant ne voyant Éric que quelques fois par année : « Pour moi, un père c'est quelqu'un qui s'implique complètement pour son enfant, qui l'élève, qui l'éduque, qui veille à ce qu'elle ne manque de rien. Là, ce n'est pas le cas. Il est le père de Nadia, dans le sens où c'est un père particulier parce qu'on est dans une configuration particulière » explique Nadine, la mère de Nadia.

Néanmoins, elle juge rétrospectivement la situation comme étant très positive pour sa fille : « Ça n'était pas prédéterminé, comme relation, et finalement, c'est assez hu- 
main. Le relationnel, on ne sait jamais comment ça va se passer. Mais l'expérience est vraiment positive. Et puis ma fille va bien, elle n'a pas de souci par rapport à ça », conclut-elle.

La deuxième configuration familiale concernée est celle de Chantale et Annabelle, dont le rôle de Fred, un ami du couple, a également évolué entre les discussions préalables au don et aujourd'hui. L'enfant (Loïc, 11 ans) appelle Fred «papa », ce qui n'était pas prévu au départ. Cela place Fred dans une situation où il craint qu'on lui reproche éventuellement son absence dans la vie de l'enfant, alors qu'il n'avait jamais prévu de s'impliquer, et ce, d'autant plus qu'il ne pense pas avoir les capacités pour assumer adéquatement un tel rôle.

Ce qui s'est passé, c'est qu'Annabelle a commencé à lui présenter comme si j'étais son papa. Le problème, c'est que moi, dès le départ, c'était clair : je ne devais pas être reconnu en tant que père. Je me retrouve dans la situation où on me dit : «Tu ne vois pas ton fils. ». Il y a un gamin qui dit : «Papa, Papa ». Même s'il est affectif, ça devient très compliqué. [...] Pour moi, un gamin, ça demande une grosse éducation, les meilleures écoles, les machins, et cetera. [...] II faut vraiment que je sois au top. Et puis, en même temps, je ne m'en pense pas capable. (Fred, donneur)

Fred confie qu'il aimerait voir l'enfant seul et avoir des contacts plus personnels en dehors de la présence de ses mères. Il exprime une certaine ambiguïté par rapport à une position de père qui n'était pas prévue au départ, mais n'est pas fondamentalement opposé à la relation que l'enfant cherche à établir avec lui. L'enfant, quant à lui, loin de ressentir l'absence de son père, témoigne plutôt de son fort attachement à son endroit. Il soutient avoir de nombreux contacts téléphoniques avec lui, parfois initiés de son propre chef, d'autres fois par Fred. Mais plus encore, Loïc témoigne de son assurance de pouvoir compter sur son père en cas de pépins : « Il a tout le temps été là. Par exemple, si j'ai un problème et que je dis que c'est urgent et je lui dis de venir, il va venir ».

Si Annabelle et Chantale perçoivent la réserve de Fred vis-à-vis de l'enfant, elles sont cependant conscientes que leur demande d'implication de sa part est plus importante que celle convenue au départ. C'est avec l'avancée en âge de Loïc que se sont précisées leurs demandes accrues d'implication envers lui, devant ce qu'elles ont perçu comme un besoin chez leur enfant. D'ailleurs, c'est ce qui explique pourquoi Annabelle n'a pas procédé à l'adoption de son fils, comme l'explique Chantal : «On ne pouvait pas fermer la porte à Loïc, plus grand, que son père puisse le reconnaître. Pour lui, ça ne change rien. Il a un papa. Ce sont des formalités administratives. Mais, voilà, on ne voulait pas fermer la porte à Fred aussi ».

\subsection{Donneur " proche "}

Dans cette catégorie, le donneur n'est pas considéré comme un père, mais bien comme un donneur, et ce, même s'il rencontre régulièrement les mères et l'enfant. Il s'agit généralement d'un ami du couple de femmes que l'enfant et les mères désignent par son prénom. Si les liens génétiques entre le donneur et l'enfant ne sont pas passés sous 
silence, il n'en reste pas moins que ce lien « ne fait pas » le père pour les personnes concernées. Deux situations illustrent bien cette catégorie.

Alain, en couple hétérosexuel, a trois enfants. Il a aidé à concevoir la cadette (deux mois) d'une famille dont nous avons rencontré l'aînée (quatre ans). La distinction opérée par Alain entre la paternité génétique et la fonction paternelle apparait clairement dans son discours, alors qu'il affirme avoir trois enfants, mais quatre descendants. Bien qu'il soit d'accord que l'enfant sache éventuellement qui il est par rapport à elle et qu'il veuille développer une relation particulière avec elle, il ne souhaite pas que cette relation soit de type père-enfant.

Ce n'est pas envisageable qu'elle m'appelle « papa ». Par contre, si elle venait sans chercher un père, mais qu'elle avait besoin de plus me connaître, savoir d'où je viens et tout ça, moi il n'y aurait pas de problème. [...] Mais si d'un coup je devais jouer un rôle dans son éducation en devenant son papa, là, ce ne sera pas prévu au départ et je pense que j'aurais besoin de tout reconsidérer avec les mamans. (Alain, donneur)

De fait, Alain prendra soin de préciser à plusieurs reprises : « Ce sont elles les parents de l'enfant. Moi, je ne me sens vraiment pas le père de cette enfant-là ». Pour ce donneur, si la famille se construit à partir des liens génétiques, l'affectivité et le partage d'une histoire commune sont essentiels pour créer des liens d'apparentement. Cette intrication des liens génétiques et affectifs comme étant à la base des liens entre apparentés est plus difficile à cerner lorsque vient le temps de déterminer les liens entre la fillette née de ses dons et ses propres enfants. Lorsque questionné à ce sujet, il soutient : " Je leur dirais [à ses enfants] : "Ce n'est pas vraiment votre petite sœur, mais en effet vous êtes à moitié du même sang”. Du coup, ça les rend un petit peu frère et sœur. Le statut est flou pour moi ».

Pour Catherine, la mère de la fillette, si ses attentes envers Alain étaient clairement de « ne pas être le parent, mais être d'accord pour répondre à des questions éventuelles de notre enfant sur sa motivation à nous aider ", elle souligne au passage l'intensité des liens qui se sont construits entre les deux familles depuis les discussions entourant le don d'Alain. Discutant des liens unissant les enfants d'Alain et Gabrielle et de sa fille, elle évoque la difficulté à les conceptualiser : « Les enfants ont un lien particulier, comme nous avons un lien particulier avec leurs parents. C'est quelque chose qui est différent de l'amitié. Ils ne sont pas frères et sœurs. Après, il n’y a pas de mots qui existent pour décrire cette relation ».

Patrick, célibataire, était un ami de Renée, en couple avec Clémentine. Il les a aidées à concevoir Corinne (six ans). Pour lui, il n'y a aucun doute : cette enfant est celle de Renée et Clémentine. Tout en étant très proche de la famille, il ne souhaite ni n'envisage aucun autre rôle que celui de donneur, et ce, d'autant plus qu'il n'a jamais caressé le souhait de devenir père. Désormais consciente de l'implication de Patrick dans sa conception, la fillette s'est mise en grandissant à l'appeler " papa », ce à quoi il a réagi en précisant clairement son rôle et en le distinguant de la paternité. 
Corinne insiste souvent et demande : «Je peux t'appeler papa ». Un jour, j'ai eu une discussion juste avec elle en lui disant : « Je suis désolé ma petite chérie. Même si tu es toute petite, je vais t'expliquer qu'il y a une différence entre un papa et un géniteur. [...] Un papa, il est là tous les jours ou la plupart du temps. [...] Tu constates bien que moi je ne suis pas là tous les jours. Toi, tu as deux mamans. Mais pour faire un bébé... ». J'en ai parlé et il se trouve que depuis ce jour, Corinne n'a jamais... elle est passée à autre chose. (Patrick, donneur)

Lorsqu'interrogée sur le rôle de Patrick dans sa vie, Corinne expliquera : « C'est un papa, mais qui n'est pas avec nous ». Elle dira l'appeler par son prénom, puisqu'« il n'aime pas qu'on l'appelle papa », mais que « Patrick aussi, ça me va ». Pour Renée, l'une des deux mères, cette distanciation relèverait d'un certain travail émotif effectué par Patrick pour éviter que ne se développe une identité paternelle, ce qu'il ne souhaite pas. Qui plus est, cela irait à l'encontre du rôle qui a été déterminé au départ entre les mères et lui quant à son implication dans la vie de la fillette. L'absence d'un terme d'adresse pouvant bien qualifier ce rôle en émergence complexifie la donne.

Il a toujours dit qu'il ne voulait pas de rôle, ce qui nous convenait. Au début, on le voyait régulièrement et on avait l'impression qu'il avait peur de créer un lien. [...] Il a un rôle un peu équivalent à un tonton. Sauf que c'était plus dur et exigeant vis-à-vis de Corinne. [...] Pour l'instant, ils se cherchent un peu tous les deux. Peut-être qu'ils finiront à trouver un mot à mettre sur leur relation qui ne soit ni «papa» ni « tonton ». (Renée, mère)

\subsection{Donneur « distant »}

Dans cette catégorie, on retrouve trois situations familiales dans lesquelles le donneur est très distant, deux d'entre eux n'étaient d'ailleurs pas connus du couple de femmes avant le don. Ces hommes sont identifiés par leur prénom ou encore, tout simplement comme «le donneur ». La distance peut être une manière de ne pas se confronter au risque de traverser la frontière entre géniteur et père, risque qui est évoqué plus ou moins clairement dans les catégories précédentes.

Alex, en couple avec un autre homme, a aidé ses amies Évelyne et Sabine à concevoir trois enfants : Théo (dix ans), Edwin (six ans) et Gaëlle (six ans). Il se considère comme sans enfants : « Je ne me considère pas du tout comme père, explique-t-il. [...] La notion d'enfant, je la rattache à la parentalité au sens de l'éducation. Avoir des enfants, c'est les élever ». Il voit la famille environ une fois par an. Il se demande d'ailleurs si la distance qu'il a instaurée n'est pas une manière de se protéger et de conserver une définition amicale de la relation avec le couple de femmes :

Ce n'est pas impossible que, de manière inconsciente, j'ai mis un peu de distance parce que je ne veux pas m'investir dans ça. [...] C'est un peu paradoxal cette distance que l'on met alors qu'on fait ça pour des gens qui nous sont proches. [...] C'est vrai aussi qu'il y a cette distance qui s'est mise parce que, pour moi, c'est aussi important que notre relation ne tourne pas qu'autour de ça. À la base, ce sont des amies avant d'être des personnes que j'ai aidées à avoir des enfants. (Alex, donneur) 
Les trois enfants parlent de lui comme étant celui « qui a donné ses graines ». Interrogé à savoir si, de ce fait, Alex serait son père, Théo explique : « Non, parce que ce n'est pas la même chose, il a juste donné des graines. Ce n'est pas lui qui s'est occupé de moi pendant que j'étais enfant ». Sa sœur abonde dans le même sens, en soutenant : « Je n'ai pas de papa, c'est un homme qui a donné la graine à une de mes mamans et qui a fait deux bébés ». Ne l'ayant vu qu'une ou deux fois (il a déménagé loin de la famille), les enfants ont peu de souvenirs du donneur. Les mères témoignent toutes deux d'une réelle affection pour ce dernier et soulignent l'importance des discussions préalables qu'elles ont eues avec lui de sorte à être tous et toutes sur la même longueur d'onde.

On s'est mis d'accord sur le fait qu'il n'aurait pas un statut de parent dans cette famille. Si les choses étaient claires pour nous, il y avait quand même des chances que les choses puissent se compliquer une fois que l'enfant serait là. On se faisait confiance pour se parler de ça mutuellement si ça devenait difficile pour les uns et pour les autres. J'avais tout à fait confiance dans le fait qu'Alex ne changerait pas d'avis, qu'il ne reconnaîtrait pas l'enfant (Évelyne, mère).

Même si elles ne craignaient pas qu'il revendique sa paternité de façon inopportune, l'adoption a quand même eu pour effet de sécuriser les mères. Pour Sabine, l'établissement de la filiation est fondamental pour la reconnaissance de sa maternité, même si, paradoxalement, ce processus qui requiert l'autorisation d'un juge, la production de divers témoignages et attestations, la rend vulnérable au jugement d'autrui : « Il y avait aussi ce truc horrible qui était de devoir prouver que je suis la mère [pendant le processus d'adoption]. Je le suis, il n'y a aucun doute dans ma tête. Mais devoir se défendre devant quelqu'un qui va nous attribuer ce titre, c'était dur », explique-t-elle.

Sophie et Julie, séparées depuis plusieurs années, ont deux enfants, Jérémie (13 ans) et Miguel (14 ans), grâce à un don de sperme de Germain que nous n'avons pas pu rencontrer. La relation du donneur avec les enfants est très distante. Il ne les rencontre pas, mais reste en contact avec Julie avec laquelle il était ami avant le don. Si l'une des mères l'identifie comme le père des enfants, sa conjointe le désigne plutôt comme géniteur. Le don s'inscrit résolument dans une aide à la procréation, sans que cela n'engage quelque relation que ce soit entre les enfants et l'homme à l'origine de leur conception, l'entourage de ce dernier n'étant d'ailleurs pas au courant de son implication dans le projet parental d'autrui : « Pour lui, c'était clair que c'était juste un don, explique Julie. Il n'en a pas parlé à sa femme », ajoute-t-elle.

Même si Germain ne voit pas les enfants, il n'en reste pas moins que son existence n'est pas oblitérée ; ils discutent de lui avec leurs mères et se montrent curieux à son endroit, mais sans insister pour le rencontrer.

Ils savent tout, oui, ils ont toujours su où il habitait, comment il s'appelait, et le pourquoi du comment. [...] Au départ [les questions portaient sur] où il habitait, comment il s'appelait, quelle était sa situation familiale, s'il avait des enfants et « Est-ce qu'on pourrait le rencontrer ? ». Ça revient périodiquement, c'est marrant. Mais ils ne sont jamais allés plus loin. Si, ils ont posé des questions comme : «Comment je l'avais rencontré [...], quelle était notre relation ». Mais, 
voilà, ils ne sont jamais allés plus loin dans leur désir de rencontre pour l'instant. (Julie, mère)

Jérémie, quant à lui, explique qu'il aimerait rencontrer Germain au moins une fois " pour voir la personne qui m'a créé ». Il dit ne pas ressentir d'abandon ni manquer de la présence de l'homme à l'origine de sa naissance, puisque ce dernier n'est pas investi d'un rôle paternel. Il affirme : «il n'a jamais été là dans ma vie, mais comme j’ai dit avant, je n'en veux pas [d'un père]. Il n'était pas là pour ça. Il a été super gentil [de faire le don], mais il n’a jamais endossé le rôle de mon père. Je ne sais pas pourquoi je l'appellerais "papa” ».

Son frère montre la même curiosité à l'endroit de son donneur, tout en réitérant le fait qu'il ne perçoit pas la nécessité de le côtoyer au quotidien : « Je ne l'ai jamais vu, explique Miguel. Et même si j'ai envie de le voir, bien c'est juste pour savoir à quoi il ressemble. Mais sinon je ne pense pas que ça apporterait grand-chose à ma vie, que je le voie ou pas ». C'est d'ailleurs précisément pour cette raison que les deux femmes ont eu recours à un donneur connu, afin que les enfants puissent un jour assouvir leur curiosité, le cas échéant. Sophie précise : «C'était surtout le fait que c'était un don et qu’il n'y avait pas d'investissement de la personne sur l'éducation et le suivi des enfants et que si, à un moment donné, les enfants voulaient le voir, ce serait possible ». Dans cette configuration, ce n'est pas tant la présence de ces hommes dans la vie des enfants qui est valorisée par l'ensemble des personnes concernées, mais bien le fait qu’ils puissent éventuellement, si le besoin se présente, aider les enfants à intégrer la question de leurs origines.

\section{Discussion}

L'ouverture de l'AMP à toutes les femmes est un enjeu âprement débattu actuellement dans l'Hexagone. Outre les oppositions déjà exprimées à l'égard du mariage pour tous, la levée des barrières d'accès pour les femmes célibataires et les couples lesbiens irait selon ses détracteurs à l'encontre du bien-être de l'enfant, en " privant » ce dernier d'un père. Ce débat se conjugue à un autre, sur la levée de l'anonymat des dons de gamètes enchâssé dans les lois de bioéthique (Mehl, 2011). Si l'anonymat des donneurs et le secret de la conception ont longtemps été la règle, ces principes sont remis en question depuis une vingtaine d'années, particulièrement depuis que les témoignages d'adultes ainsi conçus soient désormais audibles dans le discours public (Bres, 2019 ; Kermalvezen, 2008 ; Théry, Leroyer, 2014). Les psychologues responsables des consultations en clinique de fertilité soulignent d'ailleurs les dommages occasionnés par les secrets de famille (Allan, 2017; Le Lannou, 2010). En ce sens, les parents sont aujourd'hui encouragés à révéler à leurs enfants le recours à un don, même si celui-ci reste anonyme (Brunet, Kunstman, 2013).

Nos résultats montrent que les représentations des mères, des enfants et des donneurs convergent généralement vers une compréhension commune de la parenté. Chez ces familles, le donneur se situe entre deux pôles, soit à la marge du système de parenté 
en tant que tiers donneur (le don étant compris et perçu comme une simple aide à la procréation), ou pleinement reconnu comme un père, en vertu d'une vision pluriparentale de la famille. Dans quelques situations familiales, les discours des mères et des donneurs témoignent d'une certaine ambiguïté quant au rôle attendu chez le donneur en termes d'implication. Si les mères confèrent au donneur un rôle paternel (sans que cela n'aille de pair avec une officialisation de cette paternité), les hommes concernés, bien qu'endossant ce rôle auprès de l'enfant, le font à l'encontre de leur compréhension de l'entente de départ qui les situait à l'origine en périphérie de la famille. Néanmoins, on remarque que les enfants ne ressentent pas cette divergence d'opinions entre les adultes et évoluent en les considérant toutes et tous comme des figures significatives, sans que cela ne soit problématique à leurs yeux.

Le contexte sociojuridique français peut expliquer une partie de ces résultats. Tout d'abord, un homme ne peut pas échapper à la paternité légale si l'enfant ou ses mères souhaitaient faire établir une filiation paternelle. De même, un donneur peut revendiquer sa paternité, empêchant alors l'établissement d'une double filiation maternelle. Ces éventualités obligent le donneur et les mères à établir un climat de confiance, mais également à mettre en œuvre une distance adéquate qui permet d'équilibrer les définitions que chacun·e se donne de la paternité. Le climat de confiance est ainsi attesté dans certaines familles par l'amitié entre les mères et le donneur. De plus, l'adoption de l'enfant par la mère qui ne l'a pas porté permet de relativiser les risques. Que la double filiation maternelle ait été ou non établie, la mise en œuvre d'une certaine distance est rapportée par certains donneurs qui la jugent tout de même nécessaire pour établir une frontière claire entre ce qu'est un père et ce qu'est un géniteur à leurs yeux.

On peut s'étonner de l'absence de conflit entre les personnes dans ce contexte où l'entente de départ n'est pas consolidée par un encadrement législatif qui aurait pour effet de les protéger. La question de l'intrusion inopportune du donneur dans la vie des enfants de mères lesbiennes est bien documentée (Dempsey, 2012 ; Riggs, 2008). Or, comme l'ont démontré Isabel Côté et Kevin Lavoie (2016) en contexte québécois et Nicola Surtees (2017) en Nouvelle-Zélande, la négociation entre les parties préalablement au don et la clarification des attentes de part et d'autre, conjuguées à une certaine souplesse quant à la façon d'accueillir les éventuelles sollicitations de l'enfant à l'égard du donneur, présentent un gage permettant de minimiser l'émergence de conflits, ce que semble confirmer nos données. Bien que nous constations certaines divergences quant au rôle du donneur dans certaines situations - tel qu'exemplifié notamment par Annabelle, Chantal, Loïc et Fred - il apparaît néanmoins que ces divergences ne prévalent pas une coupure des liens ni d'un conflit. Néanmoins, un encadrement législatif conséquent, reconnaissant d'emblée la double filiation maternelle de l'enfant dès sa naissance, offrirait un sentiment de sécurité permettant aux femmes et aux donneurs de mettre en œuvre cette négociation de manière plus sereine.

Le vécu des familles lesboparentales qui ont recours à un donneur connu questionne sur ce qui fonde la parenté, en dépassant le découpage entre le lien biogénétique vs 
le lien électif, pour combiner ces dimensions et les redéployer dans un aménagement familial inédit (Côté, 2014). Avec les progrès des procréations médicalement assistées et l'adoption, de même que la possibilité qu'un enfant ait deux parents de même sexe depuis l'adoption de la loi du 17 mai 2013, le modèle de parenté euro-américaine décrit par David Schneider (1968) dans lequel les enfants sont nécessairement le fruit de l'union charnelle de leurs parents, s'est affaibli au profit d'une conception plus élective des liens d'apparentement, sans pour autant se frayer un chemin vers une pluriparentalité pleinement reconnue ou revendiquée (Sagnes, 2000). Le donneur est un géniteur, mais il n'est généralement pas un troisième parent.

Cette diversité des situations mesurée par la distance entre le donneur et la famille constituée des deux mères et de l'enfant met en évidence la porosité de la frontière entre la paternité et le lien génétique. Notre recherche offre ainsi une illustration de la « parenté pratique » théorisée par l'anthropologue Florence Weber (2013). À une extrémité de ce qu'on peut voir comme un continuum, le donneur est un père du quotidien, à l'autre il est un géniteur plus ou moins distant. Entre ces deux pôles, la dimension génétique va impliquer pour certaines familles que le donneur soit désigné comme père, sans pour autant occuper une place dans l'éducation de l'enfant. Ces cas de figure indiquent que les représentations sociales sont en changement et, surtout, que les personnes concernées réfléchissent à ces liens de façon créative et différente de ce qui est véhiculé par certains groupes opposés à l'homoparentalité, tout en ayant à cœur le bien-être des enfants.

\section{Bibliographie}

Allan S. (2017), Donor conception and the search for information. From secrecy and anonymity to openness, New York, Routledge.

Bres V. (2019), Je suis l'une d'entre elles. La première génération de personnes conçues par PMA avec don témoigne, Paris, L'Harmattan.

BRUNET L. (2015), « Les atermoiements du droit français dans la reconnaissance des familles formées par des couples de femmes », Enfances Familles Générations, n²3, p. 71-89.

Brunet L., Kunstman J.-M. (2013), " Gamete donation in France: the future of the anonymity doctrine », Medicine, Health and Philosophy, vol. 16, nº 1, p. 69-81.

CôtÉ I. (2014), « Du père au géniteur en passant par le tiers intéressé : représentations du rôle joué par le donneur de sperme connu dans les familles lesboparentales », Enfances Familles Générations, n²1, p. 70-95.

Côté I., LAvoie K. (2016), « De la procréation ‘assistée par’ autrui à la procréation 'négociée avec' autrui. Dialogue autour de la place du tiers donneur dans le projet parental de mères lesbiennes au Québec », Revue Quetelet, vol. 4, n 1, p. 101-121. 
Côté I., Trottier-cyr R.-P., Lavoie K., Pagé G., Dubeau D. (2019), « Récits d'enfants sur leur constellation familiale : les liens relationnels au sein des familles lesboparentales avec donneur connu au Québec », Devenir, vol. 31, n 2, p. 125-143.

DempSEy D. (2012), « More like a donor or more like a father? Gay men's concept of relatedness to children ", Sexualities, vol. 15, n² 2, p. 156-174.

Donovan D. (2000), « Who needs a father? Negotiating biological fatherhood in British lesbian families using self-insemination », Sexualities, vol. 3, n² 2, p. 149-164.

Dubeau D., Coutu S., Tremblay J.-P. (2008), « Rôles maternel et paternel. Perception des mères, des pères et des enfants » in C. Parent, S. Drapeau, M. Brousseau, è. Pouliot (dir.), Visages multiples de la parentalité, Québec, Presses de l'Université du Québec, p. 33-61.

GоLомвок S. (2015), Modern families. Parents and children in new family forms, Cambridge, Cambridge University Press.

Gross M., Courduriès J., Defederico A. (2014a), « Le recours à l'AMP dans les familles homoparentales : état des lieux. Résultats d'une enquête menée en 2012 », Sociologos, $\mathrm{n}^{\circ}$ 9. http://socio-logos.revues.org/2870

Gross M., Courduriès J., Defederico A. (2014b), « Morphologie des familles homoparentales en France en 2012 » in A. Fine, J. Courduriès (dir.), Homosexualité et parenté, Paris, Armand Colin, p. 205-212.

Herbrand C. (2018), "Ideals, negotiations and gender roles in gay and lesbian coparenting arrangements », Anthropology and Medicine, vol. 25, n 3, p. 311-328.

Kaufmann J.-C. (2011), L'entretien compréhensif, Paris, Nathan.

Kermalvezen A. (2008), Né de spermatozoïde inconnu, Paris, Presses de la Renaissance.

LECKEY R. (2014), «Infiniment plus de choses dans la vie que dans la Loi: la reconnaissance des mères lesbiennes », Droit et Société, $\mathrm{n}^{\circ}$ 86, p. 115-132.

LE LANNOU D. (2010), « Secret et anonymat du don de gamètes », Gynécologie obstétrique et fertilité, vol. 38, n 5, p. 324-331.

Malmquist A., Möllerstrand A., Wikström M., Zetterqvist Nelson K. (2014), « A daddy is the same as a mummy: Swedish children in lesbian households talk about fathers and donors », Childhood, vol. 21, n 1, p. 119-133.

Mason J., Tipper B. (2008), « Being related. How children define and create kinship », Childhood, vol. 15, n 4, p. 441-461.

Ment D. (2011), Les lois de l'enfantement. Procréation et politique en France (1982-2011), Paris, Presses de Sciences-Po.

NoRDQvist P. (2014), « Bringing kinship into being: Connectedness, donor conception and lesbian parenthood », Sociology, ${ }^{\circ}$ 48, p. 268-283.

Riggs D. (2008), « Lesbian mothers, gay sperm donors and community: Ensuring the well-being of children and families », Health Sociology Review, vol.17, n 3, p. 226-234. 
Roe A., Bridges L., Dunn J., O'connor T.G. (2006), « Young children representations of their families: A longitudinal follow-up study of family drawing by children living in different family settings ", International Journal of Behavioral Development, vol. 30, $\mathrm{n}^{\circ}$ 6, p. 529-536.

Sagnes S. (2000), « Origines ou la revanche élective » in A. Fine et C. NeIRInck (dir.), Parents de sang, parents adoptifs. Approches juridiques et ethnographiques de l'adoption, Paris, Librairie générale de droit et de jurisprudence, p. 169-183.

Samuelson M., Thernlund G., Ringström J. (1996), « Using the five field map to describe the social network of children: A methodological study », International Journal of Behavioral Development, vol. 19, n 2, p. 327-345.

SchNeider D. (1968), American kinship. A cultural account, Chicago, University of Chicago Press.

SURTEES N. (2017), Narrating connections and boundaries: Constructing relatedness in lesbian known donor familial configuration, thèse de doctorat (sociologie), University of Canterbury.

Théry I., LeRoyer A.-M. (2014), Filiation, origines, parentalité. Le droit face aux nouvelles valeurs de responsabilité générationnelle, Paris, Odile Jacob.

Weber F. (2013), Penser la parenté aujourd'hui : la force du quotidien, Paris, Éditions rue d'Ulm. 